\title{
Comparison of Image Reconstruction Algorithms in EIT Imaging
}

\author{
Benjamin Schullcke1,2, Sabine Krueger-Ziolek ${ }^{1,2}$, Bo Gong1,2, Ullrich Mueller-Lisse², Knut Moeller1 \\ ${ }^{1}$ Institute of Technical Medicine, Furtwangen University, VS-Schwenningen, Germany \\ ${ }^{2}$ Department of Radiology, University of Munich, Munich, Germany \\ Email: benjamin.schullcke@hs-furtwangen.de
}

How to cite this paper: Schullcke, B., Krueger-Ziolek, S., Gong, B., Mueller-Lisse, U. and Moelle, K. (2016) Comparison of Image Reconstruction Algorithms in EIT Imaging. J. Biomedical Science and Engineering, 9, 137-142.

http://dx.doi.org/10.4236/jbise.2016.910B018

Received: August 30, 2016

Accepted: September 20, 2016

Published: September 23, 2016

\begin{abstract}
Electrical Impedance Tomography (EIT) is a medical imaging technique which can be used to monitor the regional ventilation in patients utilizing voltage measurements made at the thorax. Several reconstruction algorithms have been developed during the last few years. In this manuscript we compare a well-established algorithm and a recently developed method for image reconstruction regarding EIT indices derived from the differently reconstructed images.
\end{abstract}

\section{Keywords}

Electrical Impedance Tomography, Ventilation Monitoring, Image Reconstruction

\section{Introduction}

Electrical Impedance Tomography (EIT) is a novel medical imaging technique which can be applied to visualize changes of impedance within in the body. Small alternating currents are injected into the body and resulting voltages are measured on the skin surface. In a clinical context EIT is used to trace changes in impedance of the lungs, caused by ventilation [1]. During inspiration the alveoli expand, which lengthens the current pathways and thus increases the impedance of the lungs [2].

Compared to well-established imaging technologies, such as X-ray computed tomography (CT) or magnetic resonance imaging (MRI), EIT has several advantages. No radiation is needed for image acquisition, which makes EIT suitable for frequent examinations or long term monitoring. Additionally, the necessary technical equipment is portable and inexpensive, which enables ventilation monitoring at the bedside.

Usually, in commercially available EIT-systems for lung imaging, an array of 16 to 32 electrodes is attached around the circumference of the chest. A pair of electrodes is 
used for current injection and the resulting voltage between the remaining electrodes is measured. Subsequently, the pair of electrodes used for current injection is changed in a rotating manner. The measured voltages are used to reconstruct images of conductivity change. The voltage measurements can be done relatively fast and enables frame rates in commercially available system of up to 50 frames/second. Thus EIT is capable of to monitoring rapid processes in the lungs, which are currently not detectable with CT or MRI.

However, a drawback of EIT is that the reconstructed image of conductivity change does not depict a thorax slice of well defined thickness, as e.g. in CT imaging. The diffuse current propagation in the thorax results in a lens-shaped volume, whose impedance changes are projected onto a two-dimensional image. As a result, impedance changes above or below the electrode plane are also reflected in the reconstructed image.

The challenge in EIT imaging is to reconstruct changes of conductivity inside a domain based on voltage measurements on the boundary of the domain. This problem is ill-posed, meaning that arbitrarily small changes in measured voltages may result in arbitrarily large values of reconstructed conductivity. The ill-posedness is usually addressed with regularization, forcing the solution, i.e. the reconstructed change in conductivity, to be either small, smooth or slowly changing.

Recently, we have developed an approach for image reconstruction including patient specific structural information (obtained e.g. from CT or MRI data) into the reconstruction process [3]. This approach facilitates the superposition of reconstructed images of conductivity change and structural images and thus provides a broader insight into the pathophysiology of the lungs.

In this paper we compare two different approaches for images reconstruction. Two EIT derived parameters, the "Center of Ventilation" $(\mathrm{CoV})$ and the "ventilation shift" (vShift) are evaluated.

\section{Methods and Material}

\subsection{Simulation Model}

Calculations in this work have been carried out using Matlab 2015a (Mathworks, Natick, USA) and the EIDORS toolbox [4]. Finite element models (FEM) were generated using NETGEN [5].

In this paper the "adjacent current stimulation pattern" was used, where currents are injected and voltages are measured between neighboring electrodes. For the considered 16 electrode system this results in 208 voltages for every frame, of which 104 are independent.

Boundary voltages for end-expiration and end-inspiration were simulated on a 3D FEM model, generated from a CT dataset. The contour of the thorax at the $5^{\text {th }}$ intercostal space was used for the outline of the model. FEM elements not-corresponding to lung tissue were assigned to a conductivity of $\sigma_{b k g}=1$. Voltages at end-expiration $\mathbf{v}_{\text {exp }} \in \mathbb{R}^{208}$ were simulated with FEM elements corresponding to lung tissue set to a 
conductivity of $\sigma_{\text {lung }}=0.3$. Voltages at end-inspiration $\mathbf{v}_{\text {insp }}$ were simulated for varying values of conductivity in the lungs:

a) Conductivity of right lung systematically varying between $\sigma_{\text {lung }}=0.3$ and $\sigma_{\text {lung }}=0.09$, with conductivity of right lung set to $\sigma_{\text {lung }}=0.09$.

b) Conductivity of dorsal lung systematically varying between $\sigma_{\text {lung }}=0.3$ and $\sigma_{\text {lung }}=0.09$, with conductivity of ventral lung set to $\sigma_{\text {lung }}=0.09$.

In this manuscript we use unit-less values for conductivity. The values for conductivity are based on the values published by Witsoe and Kinnen, whereas a collapsed lung has a conductivity of $\sigma_{\text {lung }}=141 \mathrm{mS} / \mathrm{m}$ and a conductivity of $\sigma_{\text {lung }}=43 \mathrm{mS} / \mathrm{m}$ at maximum inflation [6]. FEM elements not belonging to the lungs correspond to a conductivity of $\sigma_{\text {lung }}=480 \mathrm{mS} / \mathrm{m}$, according to the values in [7].

Figure 1(a) shows the FEM model used for simulation of $\mathbf{v}_{\text {exp }}$. Two exemplary models for the simulation of $\mathbf{v}_{\text {insp }}$ are depicted in Figure 1(b) and Figure 1(c), with lower ventilation in the right lung and the dorsal parts of both lungs, respectively.

\subsection{Image Reconstruction}

The EIT problem is usually formulated as shown in Equation (1)

$$
\hat{\mathbf{x}}=\operatorname{argmin}_{\mathbf{x}}\left\{\frac{1}{2} F(\mathbf{x})-\mathbf{z}_{2}^{2}+\lambda^{2} \mathbf{R} \mathbf{x}_{2}^{2}\right\}
$$

with $\mathbf{z}$ being the relative change in voltage, where $z_{i}=\left(v_{\text {insp }, i}-v_{\text {exp }, i}\right) / v_{\text {exp }, i}$ and i denotes the $\mathrm{i}$-th element of the vectors $\mathbf{v}_{\text {exp }}$ and $\mathbf{v}_{\text {insp }}$, respectively. Conductivity changes are denoted $\mathbf{x} ; F(\mathbf{x})$ describes the nonlinear forward model which maps changes in conductivity to voltage changes. The second them is used for regularization,

(a)

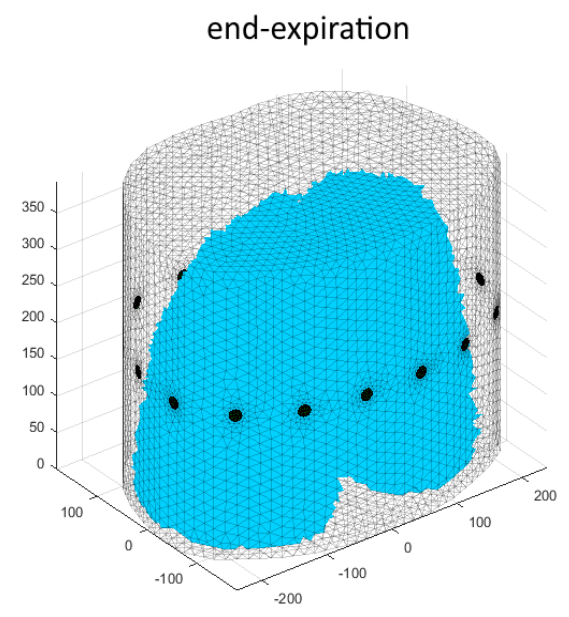

(b)

end-inspiration

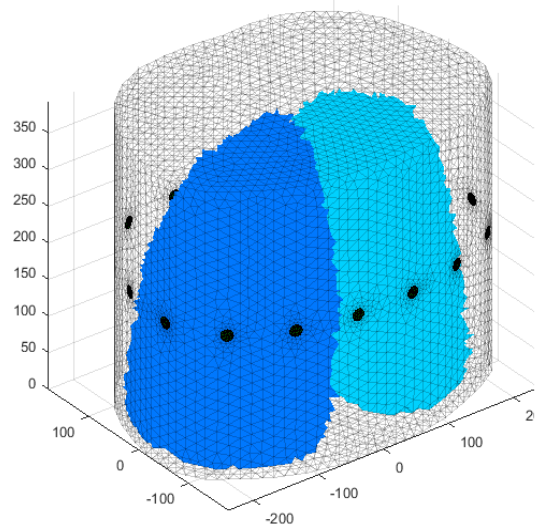

conductivity $=0.09$

conductivity $=0.3$ (c)

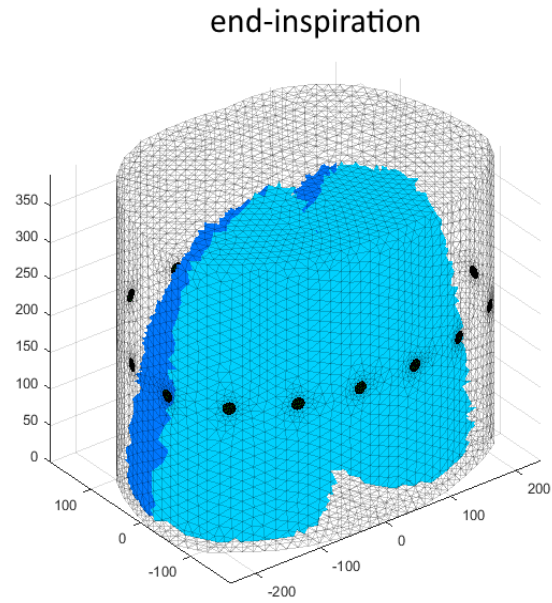

Figure 1. FEM models used for simulation of boundary voltages. 
where $\mathbf{R}$ forces the solution $\hat{\mathbf{x}}$ to be small, smooth or slowly changing and the hyperparameter $\lambda$ is used to control the amount of regularization in the solution. In this work we penalize non-smooth solutions, which means that the Laplace-Prior is used and $\mathbf{R}=\mathbf{R}_{\text {Laplace }}$.

In linearized EIT imaging the forward model is linearized around a conductivity distribution $\boldsymbol{\sigma}_{\text {ref }}$, such that

$$
F(\mathbf{x}) \approx \mathbf{J} \mathbf{x}
$$

and each element $J_{i, j}$ of the Jacobian $J$ describes the voltage change at the i-th boundary voltage induced by a conductivity change of the j-th FEM element.

Thus, for linearized EIT Equation (1) can be solved in a closed form:

$$
\hat{\mathbf{x}}=\left(\mathbf{J}^{\mathrm{T}} \mathbf{J}+\lambda^{2} \mathbf{R}\right)^{-1} \mathbf{J}^{\mathrm{T}} \mathbf{z}=\mathbf{B z}
$$

The solution of the EIT problem according to Equation (3) can be regarded as classical approach with one-step Gauss-Newton solver (one-step GN).

This reconstruction method is compared with the above mentioned approach, where patient specific morphological prior information is used in the reconstruction process. In this case the Jacobian $\mathbf{J}$ is replaced with $\mathbf{J}_{\mathrm{DCT}}=\mathbf{J} \cdot \mathbf{K}$, where the columns $\mathbf{k}_{i}$ represent certain conductivity distributions of the lungs which are based on basis vectors of a two-dimensional Discrete Cosine Transformation (DCT). A detailed description of the DCT approach can be found in [3].

\subsection{EIT Parameters}

Images were reconstructed with the one-step GN solver and with the DCT approach. For both approaches the "ventilation shift" (vShift) and the "Center of Ventilation" $(\mathrm{CoV})$ are calculated, where

$$
\text { vShift : }=\frac{\hat{\mathbf{x}}_{\text {right }}}{\hat{\mathbf{x}}_{\text {right }}+\hat{\mathbf{x}}_{\text {left }}}
$$

with $\hat{\mathbf{x}}_{\text {right }}$ denoting the reconstructed change in impedance in the right lung and $\hat{\mathbf{x}}_{\text {left }}$ respectively in the left lung.

The "Center of Ventilation" (CoV) is defined as:

$$
\mathrm{CoV}:=\frac{1}{\operatorname{sum}(\hat{\mathbf{x}})} \sum \hat{x}_{i} d_{i}
$$

with $\hat{x}_{i}$ being the reconstructed change in conductivity of the i-th FEM element and $d_{i}$ denoting the centroid position of the i-th FEM element in anterior-posterior direction. A slightly different definition of the "Center of Ventilation" has been used e.g. in [8].

\section{Results}

Exemplary reconstructions with the GN solver and the DCT approach are depicted in Figure 2 for lower ventilation in the left lung. 
Figure 3 shows vShift values and $\mathrm{CoV}$ values for both reconstruction methods. Although the reconstructed images are different, the derived vShift and CoV values show only slight differences, which is revealed in Figure 3.

\section{Discussion}

Several EIT reconstruction methods have been developed during the past years. It has already been demonstrated that indices for EIT image analysis, such as "CoV" or "vShift" are not influenced from the reconstruction method [9]. In this simulation

\section{DCT reconstruction}

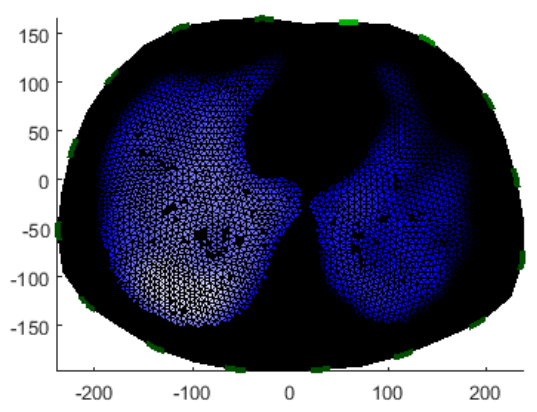

\section{GN reconstruction}

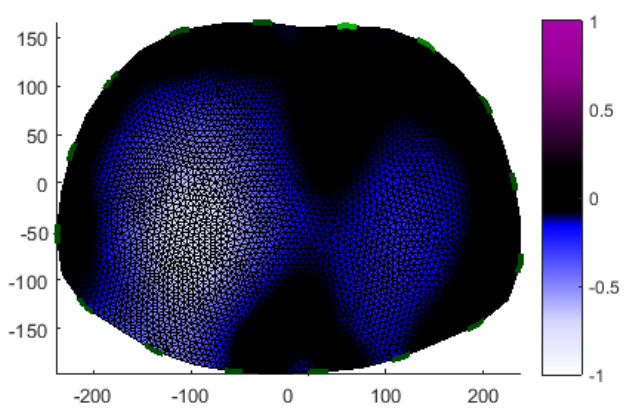

Figure 2. Left: Reconstruction of conductivity change with DCT approach. Right: Reconstruction with standard GN solver using Laplace prior.
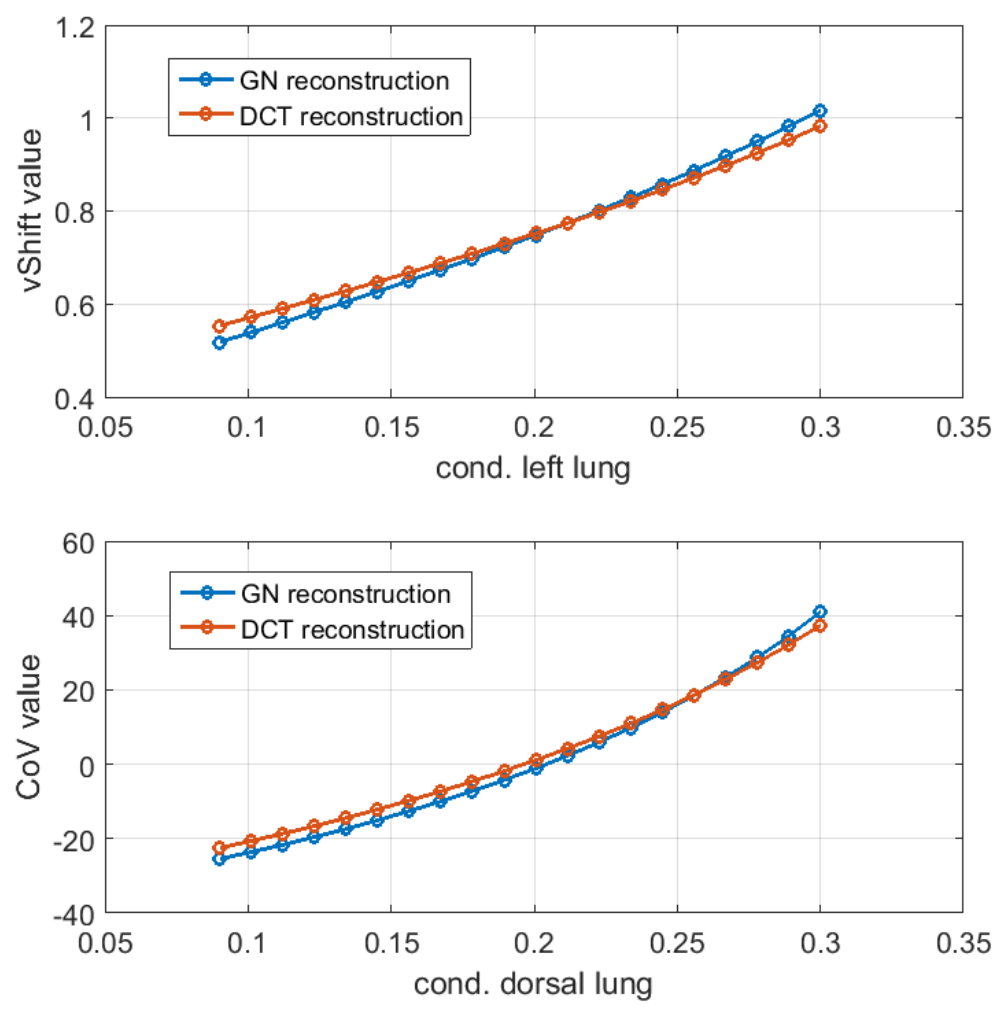

Figure 3. Top: vShift value for different reconstruction methods. Bottom: CoV value. 
study we used an algorithm including patient specific morphological information in the reconstruction process in comparison with a standard approach. Results demonstrate that both EIT indices show only slight differences for the different reconstruction methods. Reconstruction methods including morphological information might be used if the structural information is available. In patients where an actual CT or MRI dataset is not available standard EIT reconstruction algorithms, as the used one-step GN with Laplace prior still gives valuable information regarding the examined EIT indices.

\section{Acknowledgements}

This work is partially supported by the Federal Ministry of Education and Research (BMBF) under grant no. 03FH038I3 (MOSES).

\section{References}

[1] Gong, B., Krueger-Ziolek, S., Moeller, K., Schullcke, B. and Zhao, Z. (2015) Electrical Impedance Tomography: Functional Lung Imaging on Its Way to Clinical Practice? Expert Review of Respiratory Medicine, 9, 721-737. http://dx.doi.org/10.1586/17476348.2015.1103650

[2] Leonhardt, S. and Lachmann, B. (2012) Electrical Impedance Tomography: the Holy Grail of Ventilation and Perfusion Monitoring? Intensive Care Med, 38, 1917-1929. http://dx.doi.org/10.1007/s00134-012-2684-Z

[3] Schullcke, B., Gong, B., Krueger-Ziolek, S., Soleimani, M., Mueller-Lisse, U. and Moeller, K. (2016) Structural-Functional Lung Imaging Using a Combined CT-EIT and a Discrete Cosine Transformation Reconstruction Method. Scientific Reports, 6, 25951. http://dx.doi.org/10.1038/srep25951

[4] Adler, A. and Lionheart, W.R. (2006) Uses and Abuses of EIDORS: An Extensible Software Base for EIT. Physiol Meas, 27, S25-S42. http://dx.doi.org/10.1088/0967-3334/27/5/s03

[5] Schöberl, J. (1997) NETGEN An Advancing Front 2D/3D-Mesh Generator Based on Abstract Rules. Computing and Visualization in Science, 1, 41-52. http://dx.doi.org/10.1007/s007910050004

[6] Witsoe, D. and Kinnen, E. (1967) Electrical Resistivity of Lung at $100 \mathrm{kHz}$. Medical and Biological Engineering, 5, 239-248. http://dx.doi.org/10.1007/BF02474533

[7] Adler, A., Amato, M.B., Arnold, J.H., Bayford, R., Bodenstein, M., Bohm, S.H., et al. (2012) Whither Lung EIT: Where Are We, Where Do We Want to Go and What Do We Need to Get There? Physiol Meas, 33, 679-694. http://dx.doi.org/10.1088/0967-3334/33/5/679

[8] Frerichs, I., Dargaville, P.A., van Genderingen, H., Morel, D.R. and Rimensberger, P.C. (2006) Lung Volume Recruitment after Surfactant Administration Modifies Spatial Distribution of Ventilation. American Journal of Respiratory and Critical Care Medicine, 174, 772-779. http://dx.doi.org/10.1164/rccm.200512-1942OC

[9] Zhao, Z., Frerichs, I., Pulletz, S., Muller-Lisse, U. and Moller, K. (2014) The Influence of Image Reconstruction Algorithms on Linear Thorax EIT Image Analysis of Ventilation. Physiol Meas, 35, 1083-1093. http://dx.doi.org/10.1088/0967-3334/35/6/1083 
Submit or recommend next manuscript to SCIRP and we will provide best service for you:

Accepting pre-submission inquiries through Email, Facebook, LinkedIn, Twitter, etc.

A wide selection of journals (inclusive of 9 subjects, more than 200 journals)

Providing 24-hour high-quality service

User-friendly online submission system

Fair and swift peer-review system

Efficient typesetting and proofreading procedure

Display of the result of downloads and visits, as well as the number of cited articles

Maximum dissemination of your research work

Submit your manuscript at: http://papersubmission.scirp.org/

Or contact jbise@scirp.org 